\title{
4 Forschungsstrukturen
}

Verschiedenste Forschungsvorhaben auf nationaler und europäischer Ebene beschäftigen sich mit dem Problem zunehmender AntibiotikaResistenzen und gleichzeitig nachlassender Entwicklungen neuer Antibiotika.

In Deutschland werden die Forschungsaktivitäten überwiegend durch den Bund (Bundesministerium für Bildung und Forschung BMBF, Bundesministerium für Gesundheit - BMG) und die Deutsche Forschungsgemeinschaft (DFG) gefördert. Die DFG fördert zwei Sonderforschungsbereiche (SFB) und eine Forschergruppe, die sich mit der Suche nach neuen Wirkstoffen gegen Infektionserreger bzw. mit den Strukturen von Bakterien und möglichen Angriffspunkten neuer Antibiotika beschäftigen. ${ }^{88,89,90}$ Besonders gut hat sich in den letzten Jahren der Bereich der funktionellen Genomforschung entwickelt, der auch eine zentrale Rolle bei der Antibiotika-Forschung spielen wird.

Neben diesen Forschungsverbünden wurden in den vergangenen Jahren Netzwerke auf lokaler, regionaler und nationaler Ebene mit Mitteln des BMG etabliert, um Modellprojekte zu bearbeiten. Im Fokus stehen dabei beispielsweise der Antibiotika-Verbrauch und seine unterschiedlichen Auswirkungen, die Prävention von MRSA-Infektionen und die Verbesserung der Behandlung von Patientinnen und Patienten mit MRSA-Infektionen. ${ }^{91}$ Ein ähnliches Konzept wird bei der Schaffung von Gesundheitsregionen verfolgt (Beispiel: HICARE Gesundheitsregion Ostseeküste - Aktionsbündnis gegen multiresistente Bakterien). Hier sollen Management-Konzepte unter Einbeziehung aller Komponenten von der Grundlagenforschung über die klinische Intervention bis zur gesundheitsökonomischen Evaluation entwickelt werden. In Jena wurde darüber hinaus das Integrierte Forschungs- und Behandlungszentrum (IFB) Sepsis und Sepsisfolgen etabliert. ${ }^{92}$ Hier stehen eine frühzeitige effektive Diagnose und die Behandlung schwerer systemischer Infektionen im Mittelpunkt.

\footnotetext{
88 http://www.sfb630.uni-wuerzburg.de (abgerufen am: 13. April 2012).

$89 \mathrm{http} / / /$ www.uni-tuebingen.de/forschung/forschungsschwerpunkte/ sonderforschungsbereiche/sfb-766/home.html (abgerufen am: 28. August 2012). $90 \mathrm{http} / /$ www3.uni-bonn.de/forschung/forschungsprofil/forschergruppen/for-854 (abgerufen am: 28. August 2012).

91 http://www.rki.de/DE/Content/Infekt/Krankenhaushygiene/Netzwerke/ Netzwerke_node.html (abgerufen am: 09. August 2012).

92 http://www.cscc.uk-j.de/CSCC.html (abgerufen am: 13. April 2012).
} 
Die Erforschung neuer Antibiotika ist nicht nur ein Thema für bestehende Forschungsvorhaben und Netzwerke, sondern auch für das Deutsche Zentrum für Infektionsforschung (DZIF), das führende Einrichtungen der Infektionsforschung in Deutschland zusammenführt und aufeinander abstimmen soll. ${ }^{93}$ In der Konzeption des DZIF spielen auch Aspekte der Translation und translationale Strukturen eine Rolle, die sich unter anderem mit der Suche nach neuen Wirkstoffen befassen.

Auf europäischer Ebene fördert die Innovative Medicines Initiative (IMI) als öffentlich-privater Verbund - beteiligt sind die Europäische Kommission und der europäische Dachverband der pharmazeutischen Industrie - die Suche nach neuen Wirkstoffen und antimikrobiellen Substanzen. ${ }^{94} \mathrm{Im}$ Rahmen des 6. EU-Forschungsrahmen programms befassten sich Projekte mit der Antibiotika-Resistenz-Problematik. ${ }^{95}$ Der bis Ende August 2012 laufende Forschungsverbund ERA-NET PathoGenoMics, ein Konsortium aus neun Ländern, untersuchte molekulare Mechanismen der Pathogenität von Mikroorganismen, entwickelte neue Diagnostika und analysierte epidemiologische Zusammenhänge bei der Ausbreitung pathogener Mikroorganismen. Führende europäische Forschungseinrichtungen haben ihre Kompetenzen im Network of Excellence (NoE) EuroPathoGenomics gebündelt. ${ }^{96}$ An der Joint Programming Initiative „The Microbial Challenge - An Emerging Threat to Human Health“ beteiligen sich 15 Staaten. Im Rahmen dieser Initiative untersuchen Wissenschaftler die Biologie und Dynamik von Resistenzen, erarbeiten Strategien zur Prävention von Resistenz-Entwicklung und evaluieren innovative Behandlungsmöglichkeiten. Verbünde im 7. EU-Forschungsrahmenprogramm bearbeiten ein breites Spektrum wissenschaftlicher Probleme hinsichtlich der Entstehung und Ausbreitung von Antibiotika-Resistenzen, suchen nach neuen Antibiotika und Targets für eine Antibiotika-Therapie sowie nach schnelleren und leistungsfähigeren Diagnostikverfahren. ${ }^{97}$ Ausschreibungen im Arbeitsprogramm 2013 des 7. EU-Forschungsrahmenprogramms knüpfen an die 2011 veröffentlichte Resolution der EUKommission zur Antibiotika-Resistenz an.

Auch einige kleinere und mittlere Unternehmen (KMU) sind in der Antibiotika-Entwicklung aktiv und erfolgreich tätig. Die EU und ebenso

93 http://www.bmbf.de/de/16544.php (abgerufen am: 13. April 2012).

$94 \mathrm{http}: / /$ www.imi.europa.eu/ (abgerufen am: 09. August 2012).

95 http://cordis.europa.eu/projects/home_en.html (abgerufen am: 30 August 2012).

96 http://www.noe-epg.uni-wuerzburg.de/ (abgerufen am: 09. August 2012).

97 http://cordis.europa.eu/fp7/home_en.html (abgerufen am: 27. April 2012). 
das BMBF fördern die Antibiotika-Forschung im Rahmen von Programmen, an denen auch KMU beteiligt sind.

Trotz der bereits existierenden Forschungsstrukturen ist mit Blick auf die Entwicklung neuer Antibiotika eine stärkere Förderung notwendig, welche es ermöglicht, Forschungsstrukturen über nationale Grenzen hinweg längerfristig zu etablieren. Insbesondere sollten die Projekte der letzten Jahre evaluiert und gute Ansätze weiter verfolgt werden. 
